\title{
ADAPTATION IN MICRO-ORGANISMS
}

\begin{abstract}
A SYMPOSIUM on "Adaptation in Micro-organisms" was held by the Society for General Microbiology during April 14-15 at the Royal Institution, London. The papers of the contributors had been circulated to members of the Society beforehand, and the symposium was arranged in the form of discussions of each paper following a brief introduction by the author.

Dr. R. Y. Stanier (University of California), who introduced the symposium, defined two types of adaptive phenomena. Evolutionary adaptation involves selection of an organism possessing suitable characteristics which enable it to multiply in a specific environment and to survive natural environmental changes. In physiological adaptation, cells of a population adjust themselves directly to meet a sudden change in the environment. Classical genetical adaptation of micro-organisms is restricted, he suggested, by the predominance of haplophase and " $a$ spotty distribution of sexuality", although this latter characteristic was questioned in discussion by Dr. Muriel Robertson and Dr. G. Pontecorvo. Yet micro-organisms live in micro-environments which are subject to rapid fluctuations- the environments are "discontinuous in time". We might expect, therefore, physiological adaptation to be of considerable importance among microbial populations.
\end{abstract}

Dr. A. C. R. Dean and Sir Cyril Hinshelwood (University of Oxford) discussed further the distinction between adaptations which could be attributed to mutant selection and those which are based upon ahanges in the whole population. They have evidence that the ability of Bacterium lactis aerogenes (Aerobacter aerogenes) to grow in the presence of proflavine or chloramphenicol is not due to selection of drug-resistant strains. For example, cultures derived from colonies which have grown in the presence of high concentrations of proflavine show no greater resistance to proflavine than does the original culture which has never come in contact with the drug. Resistance has not been deeply impressed on the organism, neither has a mutant been selected. However, resistance which is not lost on growth in the absence of the drug may be impressed by continuous sub-culture in the presence of the drug. That this observation could be due to mutant selection seemed to Dr. Dean and Sir Cyril to be incompatible with the high reversion-rate which has to be assumed to account for the stability of the non-resistant culture. They concluded that the selection of mutant strains might not play a big part in the development of drug resistance.

Dr. A. W. Ravin (Columbia University, New York) discussed the occurrence in populations of Aerobacter aerogenes of cells with varying ability to utilize citrate and other intermediates of the tricarboxylic acid cycle. Normally, growth of the organism on these compounds occurs after a lag during which nearly all the population develop the ability to utilize these acids. This ability is lost on subculture on glucose, but is regained on further subculture on citrate. In any culture of the organism, however, Dr. Ravin has shown that there are some mutant cells which do not utilize citrate; their relative number is increased by irradiation with ultra-violet light. Cultures derived from such cells show a permanent loss of ability to utilize citrate.
It was pointed out that heterogeneous populations probably occur very frequently in microbiology and may complicate studies of adaptation. In discussion Dr. Monod suggested that such heterogeneity of a population might be due to some cells having, and others lacking, energy reserves or intermediates of some kind necessary for synthesis of the adaptive enzyme required for growth. Later in the symposium, Dr. M. R. Pollock followed up this point of an overall shortage of some such compound, speaking of the "quantum mechanics of bacteria".

Dr. Ravin also introduced the paper of Dr. P. P. Slonimski (Laboratoire de Génétique du Centre National de la Recherche Scientifique, Paris), who was unable to be present. Dr. Slonimski's work on the effect of acriflavine on the formation of cytochrome oxidase by yeast provides an interesting example of a specific relation between the hereditary and non-hereditary nature of formation of an enzyme system. Yeast cells which have been grown in the presence of acriflavine give rise to small colonies. Cells derived from such colonies are deficient in cytochrome oxidase and retain this characteristic indefinitely during vegetative reproduction. Acriflavine also specifically inhibits the adaptive formation of cytochrome oxidase and related enzymes in anaerobically grown yeast when the cells are exposed to oxygen. The experimental results indicate that acriflavine inhibits some specific reaction which governs both the hereditary and the adaptive mechanisms of respiratory enzyme formation.

Other papers on the first day dealt with the mechanism of synthesis of adaptive enzymes. The biosynthesis of adaptive, as opposed to constitutive, enzymes can be studied directly, and it seems reasonable to believe that information derived from such studies should be applicable to the formation of constitutive enzymes and of proteins in general.

Dr. M. R. Pollock (Institut Pasteur, Paris, and National Institute for Medical Research, London) spoke of the difficulty of studying adaptive enzyme synthesis as an isolated phenomenon, since it requires such close integration of energy-yielding reactions and reactions yielding intermediates in the synthetic chain. The adaptive formation of penicillinase by Bacillus cereus has been investigated in cells during the logarithmic phase of growth when metabolism has reached a steady state. Brief exposure of the cells to penicillin at this stage induces formation of the enzyme, which continues after removal of the drug. Quantitative considerations indicate that the ratio of molecules of penicillin fixed to molecules of penicillinase formed is very small, suggesting a catalytic role for penicillin, probably as a complex with a cell component. Dr. Pollock visualized a cyclical chain of reactions primed by an inducer-cell component complex and leading to the formation of the enzyme.

The role of the inducer in enzyme synthesis was further discussed by Dr. M. Cohn and Dr. J. Monod (Institut Pasteur, Paris). They suggested that induction is necessary in the formation of both constitutive and adaptive enzymes. In the former case the specific inducer may be a product of the metabolism of normal cells, whereas an external inducer is required for the creation of an adaptive system. It is postulated that either external or internal inducers 
give rise to organizers which are concerned in the arrangement of amino-acids into enzyme proteins. Thiophenol- $\beta$-galactoside is apparently a competitive antagonist of induced $\beta$-galactosidase synthesis in Baclerium coli, and studies have been commenced in an effort to find compounds which may act as specific antagonists of inducers of constitutive enzymes.

Dr. S. Spiegelman and Dr. H. O. Halvorson (University of Illinois) have investigated the utilization of free amino-acids in cells of Saccharomyces cerevisioe during the formation of the adaptive system maltozymase. Enzyme synthesis occurs at the expense of the free amino-acids within the cells and cannot occur if the cells contain no free aminoacids. Their results suggest that the first stable (an important qualification which was sometimes overlooked in discussion) intermediates in enzyme formation must be of considerable complexity.

Prof. R. Knox (Guy's Hospital Medical School, London) emphasized differential temperature sensitivity as a means of distinguishing between adaptive processes and growth not involving such mechanisms. The formation of adaptive enzymes appears to be more sensitive to temperature change than is cell proliferation. For example, the sensitivity of Bacillus cereus to penicillin is increased at temperatures which suppress the adaptive formation of penicillinase, but which do not affect growth in the absence of the drug. The sensitivity of adaptive changes to heat may be of importance in chemotherapy; there is already some evidence that isoniazide is more effective against tubercular infection in animals with abnormally high body temperatures.

On the second day Dr. E. P. Abraham (University of Oxford) ably introduced a discussion on drug resistance among micro-organisms. The development of resistance has most frequently been observed in cultures which have been in contact with the drug. In some cases selection of a spontaneous mutant may occur ; in others, growth in the presence of the drug seems to be due to adaptive changes in most of the cells of the population. Dr. Abraham considered it very difficult to determine which type of change has occurred and found the replica technique of Lederberg and Lederberg alone convincing in its ability to make this distinction. Finally, he pointed out that it is scarcely surprising that so little is known about the factors causing drug resistance, when the modes of action of so few drugs have been elucidated.

It was evident from the following discussions that organisms differ greatly in their ability to tolerate any one drug, and that even in a single strain different types of resistant variant may be isolated in a single experiment.

This was particularly emphacized by Dr.Mary Barber (St. Thomas's Hospital Medical School, London), who showed slides illustrating the wide variation, both heritable and non-heritable, among strains of Staphytococcus aureus which can grow in the presence of antibiotics.

Dr. D. A. Mitchison (Postgraduate Medical School, London) discussed the variations in resistance found among organisms isolated from hosts after streptomycin and isoniazid therapy. Changes in the population of tubercle bacilli have been noted in cases treated with streptomycin; drug-resistant strains may be replaced by sensitive strains on withdrawal of the drug. He stressed the importance of this phenomenon and also the relation of resistance to virulence and viability in medicine and public health.
The concluding papers dealt with a variety of micro-organisms ranging in size from bacteriophage to Paramecia. Dr. L. F. Hewitt (Serum Research Institute, Surrey) discussed the control that bacteriophages may exert over evolution in bacteria. They are known to act first by selection of phage-resistant strains due to lysis of phage-sensitive cells, and, secondly, by the actual transmission of genetic characters of one cell to another through an infecting phage entering a cell without lysis occurring. In the second case detection is difficult since it depends upon contact of the phages with organisms sensitive to lysis by them. In addition, bacteriophages are themselves capable of adaptation. Thus from one phage active against a particular strain of Corynebacterium diphtherioe, phages can be obtained which lyse cells of different serological type.

Many of the arguments concerning the nature of changes in bacterial populations have centred on the question of whether or not a mutation has occurred. Dr. G. H. Beale (University of Edinburgh) pointed out that it is impossible to provide unequivocal answers to such questions since controlled breeding experiments cannot be carried out with bacteria. In Paramecium, however, such experiments can be performed. He discussed several types of adaptation in this organism, all of which arose during asexual reproduction. In the case of variation in antigenic structure, it was shown that a change in the eytoplasm had occurred rather than a gene mutation followed by seleation. Similar phenomena probably account for other adaptations studied in this organism. Nevertheless, the ability of a strain to adapt to given conditions is genetically controlled.

Dr. L. F. L. Clegg (National Institute for Research in Dairying, Shinfield) and Dr. S. E. Jacobs (Imperial College of Science and Technology, London) described work with thermophilic organisms which grow optimally at temperatures above $50^{\circ} \mathrm{C}$. Though most thermophiles can adapt themselves to grow at lower temperatures, there are very few examples of adaptation of a mesophilic organism to the thermophilic mode of life. Dr. M. R. Pollock suggested that the inability of some thermophiles to grow at lower temperatures might reside in changes in the equi librium of reactions concerned in synthetic processein the cell.

Prof. W. Brown and Dr. R. K. S. Wood (Imperial College of Science and Technology, London) dealt in particular with the adaptation of fungi to their natural habitat. Little is known of the physiological processes which enable them to flourish in the chosen conditions. The life-cycle of fungi is a very important factor in their ability to survive wide environmental fluctuations. The vegetative stage gives rise quickly to conidia, which can germinate immediately when favourable conditions arise. Under unfavourable conditions, fungi can carry on sexual reproduction which yields long-lived resistant spores.

Perhaps the most striking impression gained from the symposium as a whole was the very considerable measure of agreement over the respective parts played by single cell and selective adaptation in microbial growth. Much of the lively discussion appeared to arise through the inability of chemists, bacteriologists and geneticists to appreciate fully one another's modes of expressing their conclusions. It was also clear that even a surface understanding of the closer physiological relationship between the two mechanisms of adaptation must await further experimental work. 